\section{Penoscrotal Extramammary Paget's Disease with Multiple Lymph Node Metastasis}

\author{
Yoon Taek Koo, Kyung Won Minn, Hak Chang \\ Department of Plastic and Reconstructive Surgery, Seoul National \\ University Hospital, Seoul National University College of \\ Medicine, Seoul, Korea

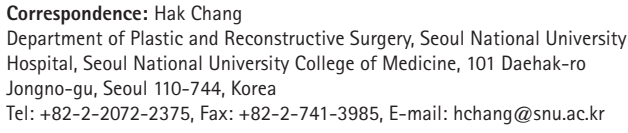

Extramammary Paget's disease (EMPD) is an uncommon intraepithelial adenocarcinoma. EMPD in the penis and scrotum, first described in 1888, is extremely rare [1]. EMPD is a rare skin disease that is limited to the epidermis, but has the potential to invade the dermis and metastasize. If dermal invasion has occurred, the rate of lymph node or distant metastasis will increase [2]. The rate of lymph node metastasis is reported to be $26 \%$ in patients with invasive primary lesions [3]. EMPD might be associated with an increased risk of underlying internal malignancy of the lower gastrointestinal or genitourinary tract. We

Fig. 1.

Preoperative view: a 69-yearold male patient with penoscrotal extramammary Paget's disease. describe a case of penoscrotal extramammary Paget's disease with multiple lymph node metastasis.

A 69-year-old man was referred to us with a oneyear history of a painless erythematous skin rash on the penis and scrotum (Fig. 1). He first visited a local clinic and was treated with topical agents. Despite conventional treatment, the lesion extended to the scrotum and penis. The patient initially underwent a punch biopsy and the pathology report came back as EMPD. On physical examination, there was a 2-cm round, firm mass in the left inguinal area. A computed tomography (CT) scan of the abdomen revealed a $2.1-\mathrm{cm}$ round peripheral rim-enhancing lymph node (LN) in the left inguinal area and a $2.8-\mathrm{cm}$ enlarged ovoid lymph node in the right inguinal area (Fig. 2). Ultrasound-guided biopsy of the localized mass in the left inguinal area confirmed the diagnosis of metastatic carcinoma.

Before wide excision of the scrotal and penile lesion, mapping biopsies were performed to determine the exact margins of the lesion. However, during the preoperative period, the mass of the right inguinal area increased from 2.8 to $3.58 \mathrm{~cm}$. Therefore, the patient was admitted to the department of oncology and had an extensive cancer work-up. The tumor markers were within normal range: alpha-fetoprotein $1.5 \mathrm{ng} / \mathrm{mL}$ (normal range, 0-20 ng/mL), carcinoembryonic antigen $2.6 \mathrm{ng} / \mathrm{mL}$ (normal range, $0-5 \mathrm{ng} / \mathrm{mL}$ ), and prostate-specific antigen $0.712 \mathrm{ng} / \mathrm{mL}$ (normal range, 0-3 ng/mL). Routine colonoscopy and esophagogastroduodenoscopy were performed to check for internal malignancy of the lower gastrointestinal track. However, there was no evidence of an underlying internal malignancy. A whole-body positron emission tomography (PET)/CT scan revealed metastatic lymphadenopathy on both inguinal areas and the left external iliac space. No other abnormal hypermetabolic lesion was found (Fig. 3).

After the full cancer work-up, the patient underwent wide local excision with both inguinal and left external iliac lymphadenectomy. We performed surgical wide excision of a $16.5 \mathrm{~cm} \times 8.5 \mathrm{~cm}$ lesion of the scrotum and penis. The skin defect was covered with a scrotal flap and split-thickness skin graft for the penile shaft.

The pathologic findings with immunohistochemical staining revealed extramammary Paget's disease (Fig. 4), measuring $7 \mathrm{~cm} \times 4.2 \mathrm{~cm}$. Moreover, a $1.5 \mathrm{~cm}$ $\times 1 \mathrm{~cm}$ dermal invasion lesion of the penis and scrotum was seen (Fig. 5).

All of the surgical safety margins were clear. In this 
report, our data showed that 17 of 20 lymph nodes showed metastasis. The lymph node metastasis was expressed in $75 \%$ (3/4) in the right inguinal, $85.7 \%$ $(6 / 7)$ in the left inguinal, $100 \%(6 / 6)$ in the left external iliac, and $66.6 \%(2 / 3)$ in the peri-iliac vessel.

An abdomino-pelvic CT scan was performed 1 month postoperatively. Based on the CT scan data, a $1.8 \mathrm{~cm} \times 2.5 \mathrm{~cm}$ lymphocele was found in the left external iliac and inguinal region. However, there was no evidence of metastasis in the abdomen or pelvis. The patient received $45 \mathrm{~Gy} / 25 \mathrm{Fx}$ postoperative radiotherapy. He was followed up in the outpatient department for 6 months without any evidence of recurrence or other complications.

EMPD is a rare, slow-growing intraepithelial lesion with well-defined moist erythematous or white plaques. Complete surgical excision is the treatment of choice for EMPD. Mohs micrographic surgery or mapping biopsy is useful for determining the normal skin margin for wide excision. Non-surgical treatments, such as radiation therapy, topical chemotherapy with imiquimod, and photodynamic therapy with topical aminolevulinic acid are effective in inoperable patients. Only radiation therapy can be applied for deep lesions such as lymph node metastasis. Several authors have reported on EMPD patients treated with radiation therapy at total doses of 30 to $54 \mathrm{~Gy}$ in 3 to 25 fractions. However, the optimal radiation dose for treatment of a metastatic lymph node is controversial [4].

EMPD rarely invades the dermis or spreads to the local inguinal lymph nodes or remote sites; only a few such cases have been reported. Chan et al. [2] reported that dermal invasion was found in $18.8 \%$ on histological examination after wide excision. Regional lymph node metastasis was found in $14.5 \%$, and there was a statistically significant relationship between dermal invasion and regional lymph node metastasis. In cases of primary invasive tumor without clinical lymph node metastasis, elective lymph node dissection has been recommended [3]. Our case showed a focal dermal invasion in the primary lesion with bilateral inguinal and left external iliac LN metastasis. The standard treatment for advanced EMPD with distant metastasis is controversial. We performed successful wide excision, obtaining clear margins, and multiple lymphadenectomy. We applied additional radiation therapy because most of the pathologic findings of the lymph nodes were positive. There have been some reports about the clinicopathology and prognosis of metastatic EMPD [5]. However, they have not been well established, so further clinical follow-up is needed.

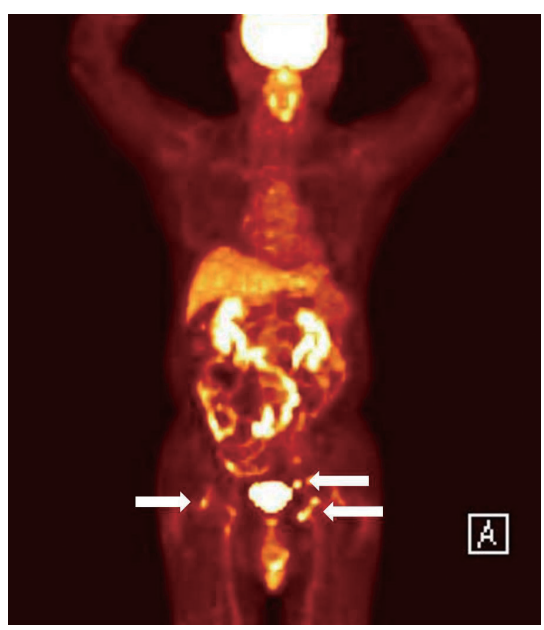

Fig. 3.

A full body positron emission tomography computed tomography scan showed metastatic lymphadenopathy in both inguinal areas and the left external iliac space (white arrows).

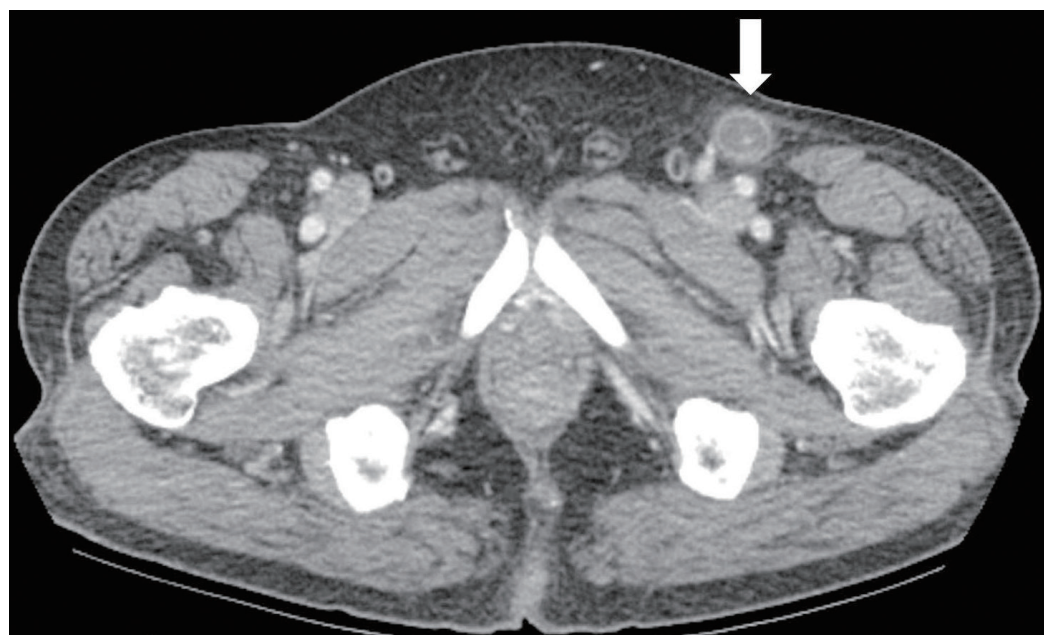

Fig. 2.

A computed tomography scan of the abdomen revealed bilateral inguinal lymph node metastasis. The white arrow indicates a $2.1-\mathrm{cm}$ round peripheral rimenhancing lymph node in the left inguinal area. 


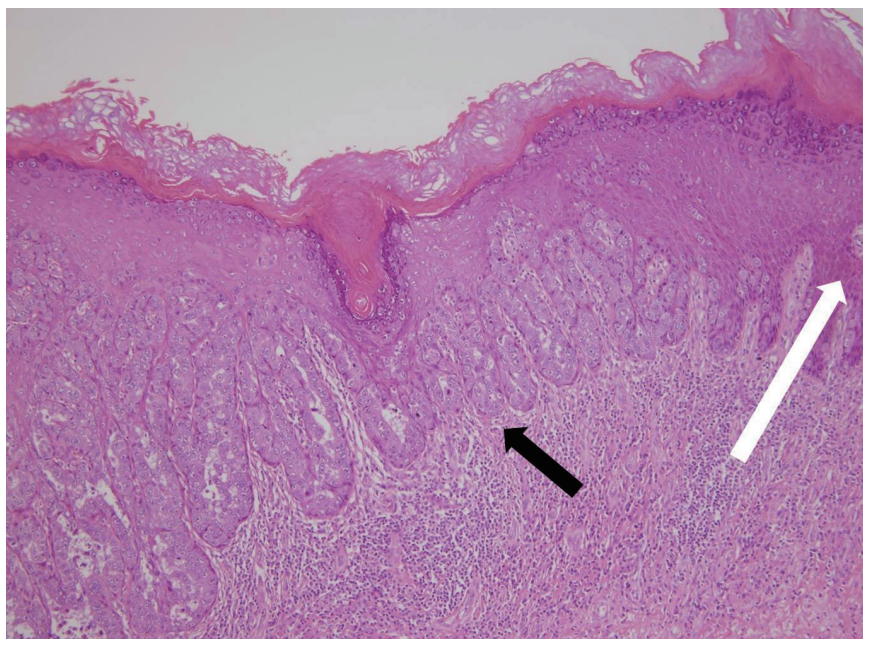

Fig. 4.

Histological findings. The black arrow indicates Paget cells with atypical round nuclei with abundant pale granular cytoplasm. The white arrow indicates normal cell lining $(H \& E, \times 100)$.

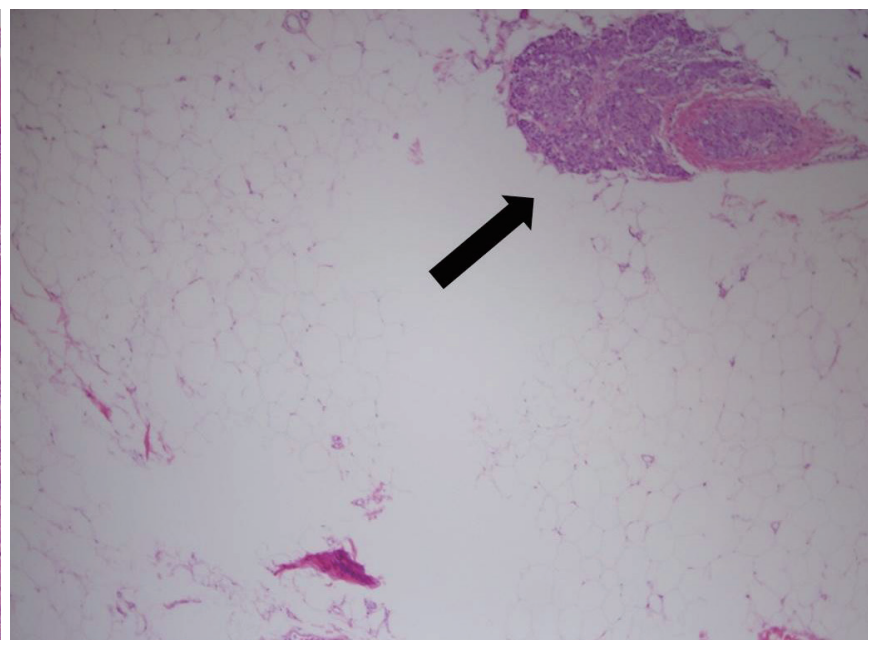

Fig. 5. Histological findings. The black arrow indicates a tumor cell nest in the subcutaneous layer $\left(H \& E_{1} \times 20\right)$.

\section{References}

1. Crocker HR. Paget's disease affecting the scrotum and penis. Trans Pathol Soc Lond 1888-1889;40:187-91.

2. Chan JY, Li GK, Chung JH, et al. Extramammary Paget's disease: 20 years of experience in chinese population. Int J Surg Oncol 2012;2012:416418.

3. Tsutsumida A, Yamamoto Y, Minakawa H, et al. Indications for lymph node dissection in the treatment of extramammary Paget's disease. Dermatol Surg 2003; 29:21-4.

4. Brierley JD, Stockdale AD. Radiotherapy: an effective treatment for extramammary Paget's disease. Clin Oncol (R Coll Radiol) 1991;3:3-5.

5. Zhu Y, Ye DW, Yao XD, et al. Clinicopathological characteristics, management and outcome of metastatic penoscrotal extramammary Paget's disease. Br J Dermatol 2009;161:577-82.

\section{Use of a Silicone Gel Sheet Vaginal Mold in McIndoe Vaginoplasty}

Sang Wha Kim ${ }^{1}$, Dong Yeon $\mathrm{Kim}^{1}$, Deuk Young $\mathrm{Oh}^{1}$, Jung Ho Lee ${ }^{1}$, Jong Won Rhie ${ }^{1}$, Sang Tae Ahn ${ }^{1}$, Joo Hee Yoon ${ }^{2}$

Departments of ${ }^{1}$ Plastic Surgery and ${ }^{2}$ Obstetrics and Gynecology, Seoul St. Mary's Hospital, The Catholic University of Korea, Seoul, Korea

\section{Correspondence: Deuk Young Oh}

Department of Plastic Surgery, Seoul St. Mary's Hospital, The Catholic University of Korea, 222 Banpo-daero, Seocho-gu, Seoul 137-701, Korea Tel: +82-2-2258-6143, Fax: +82-2-594-7230

E-mail: ohdeuk1234@hanmail.net

\section{This article contains supplemental Video S1.}

This research was supported by Seoul St. Mary's Clinical Medicine Research Program (2011) through The Catholic University of Korea.

No potential conflict of interest relevant to this article was reported.

Received: 3 Apr 2013 • Revised: 20 Jun 2013 • Accepted: 2 Jul 2013 pISSN: 2234-6163 • elSSN: 2234-6171

http://dx.doi.org/10.5999/aps.2013.40.5.652 • Arch Plast Surg 2013;40:652-655 Copyright (C) 2013 The Korean Society of Plastic and Reconstructive Surgeons This is an Open Access article distributed under the terms of the Creative Commons Attribution Non-Commercial License (http://creativecommons.org/licenses/by-nc/3.0) which permits unrestricted non-commercial use, distribution, and reproduction in any medium, provided the original work is properly cited.

The McIndoe operation is a widely performed surgical procedure for neovaginal reconstruction [1]. It creates a neovaginal cavity by dissecting between the bladder and rectum, and grafts skin to resurface a surgically created neovagina. A space-occupying vaginal mold is required to maintain the skin graft. An unsuitable 